\title{
De novo design, solution characterization, and crystallographic structure of an abiological Mn-porphyrin binding protein capable of stabilizing a Mn(V) species
}

\author{
Samuel I. Mann $\$$, Animesh Nayak§, George T. Gassner"l, Michael J. Therien`, William F. DeGrado*, \\ tDepartment of Pharmaceutical Chemistry and the Cardiovascular Research Institute, University of California at San Fran- \\ cisco, San Francisco, California 94158-9001, United States

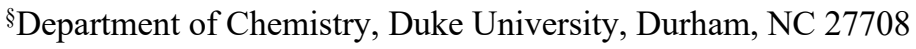 \\ "Department of Chemistry and Biochemistry, San Francisco State University, San Francisco, California 94132, USA.
}

\begin{abstract}
De novo protein design offers the opportunity to test our understanding of how metalloproteins perform difficult transformations. Attaining high-resolution structural information is critical to understanding how such designs function. There have been many successes in the design of porphyrin-binding proteins, however crystallographic characterization has been elusive, limiting what can be learned from such studies as well as the extension to new functions. Moreover, formation of highly oxidizing highvalent intermediates poses design challenges that have not been previously implemented: 1) purposeful design of substrate/oxidant access to the binding site and 2) limiting deleterious oxidation of the protein scaffold. Here we report the first crystallographically characterized porphyrin-binding protein that was programmed to not only bind a synthetic Mn-porphyrin but also maintain binding site access to form high-valent oxidation states. We explicitly designed a binding site with accessibility to dioxygen units in the open coordination site of the Mn center. In solution, the protein is capable of accessing a high-valent Mn(V)-oxo species which can transfer an $\mathrm{O}$-atom to a thioether substrate. The crystallographic structure is within $0.6 \AA$ of the design, and indeed contained an aquo ligand with a second water molecule stabilized by hydrogen-bonding to a Gln sidechain in the active site, offering a structural explanation for the observed reactivity.
\end{abstract}

\section{INTRODUCTION}

Hemes are one of the most widely used and versatile cofactors employed in biology. ${ }^{1-3}$ Nature has evolved a variety of heme-binding protein scaffolds to perform a broad range of functions, such as ligand sensing and transport, electron transfer, protection from reactive oxygen species, and substrate oxidation. Free heme, without the aid of a protein scaffold, is prone to aggregation and has drastically reduced functionality. On the other hand, synthetic chemists have developed a massive library of metalloporphyrins to recapitulate the reactions performed in nature (albeit often less selectively and at slower rates) but also to perform reactions not found in Nature: such as cyclopropanation and amination. ${ }^{2,4-5}$ While directed evolution has produced engineered heme proteins with impressive rates and selectivities for abiological reactions ${ }^{6-7}$, de novo protein design offers the opportunity to test our understanding of protein structure-function relationships..$^{8-12}$ A complete understanding of how proteins tune the function of heme cofactors combined with novel reactivity of synthetic cofactors, would allow for the design of functional metalloproteins that can expand upon the scope of natural proteins. ${ }^{10}$

The de novo design of metalloproteins has seen many successes, ${ }^{8-10,13}$ but the ability to design cofactor-binding proteins with sub- $\AA$ accuracy, without the need for evolution or experimental optimization of the scaffold, has only recently been achieved. ${ }^{14}$ De novo heme-binding helical bundles have been designed from first principles, including maquettes $^{10,15-16}$ and binary-patterned artificial superfamilies, ${ }^{17}$ however, these have not been successfully structurally characterized at high resolution. Although they have shown the ability to tune redox potentials of heme cofactors and can perform a limited range of peroxidase chemistry, ${ }^{15,17-20}$ maximizing the utility of these designed heme-binding proteins through further design refinements has been limited by the inability to acquire high-resolution structural information providing function without structure. In an effort to address this issue of structural non-uniqueness, our group employed a strategy used by natural cofactor-binding proteins: a four-helix bundle was computationally designed to bind a photo-reducible $\mathrm{Zn}$ porphyrin $\left(\left(\mathrm{CF}_{3}\right)_{4} \mathrm{PZn}\right)$ (Fig. 1\&2) adjacent to a wellpacked distal core to ensure a well-packed, structurally unique protein. This led to a high-resolution NMR structure of PS1 showing sub- $\AA$ accuracy compared to the design. However, this work was conducted using $\mathrm{Zn}$ (II) porphyrin, selected for photophysical applications, and therefore containing a redoxinactive metal ion. ${ }^{14}$

Here we explore and extend the principles used in the design of PS1 to create a non-natural manganese diphenylporphyrin-binding protein that was crystallographically characterized; providing us with information that is lacking in solution NMR: the exact positioning of the metal ion relative to the protein and the location of aqua ligands and associated water molecules within the binding site.

A common feature in many heme enzymes and synthetic model complexes is the binding of a dioxygen species $\left(\mathrm{O}_{2}\right.$ or $\left.\mathrm{H}_{2} \mathrm{O}_{2}\right)$ which is then heterolytically cleaved to make a high-valent metal-oxo species with the release of water. ${ }^{2-4}$ Thus, the ability to accommodate two O-atom units (e.g., one 
ligated and one distal water molecule) is critical to facilitating oxidative chemistry. Additionally, it is essential to be able to access high-valent intermediates without deleterious effects to the protein or cofactor. We achieve these enabling features for the first time in a de novo designed protein. Herein we report the computational design of the first crystallographically characterized de novo metalloporphyrin-binding protein.

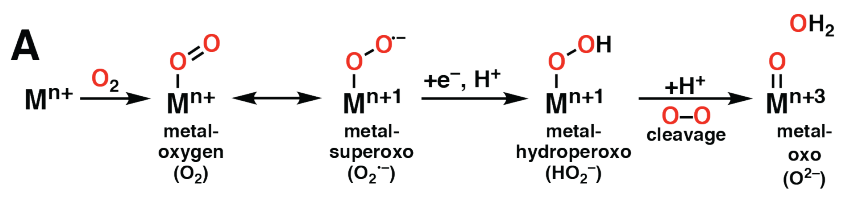

B

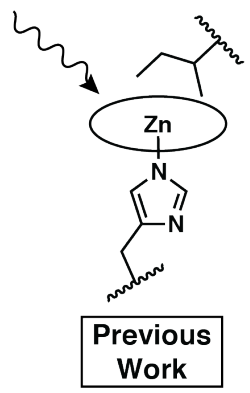

C

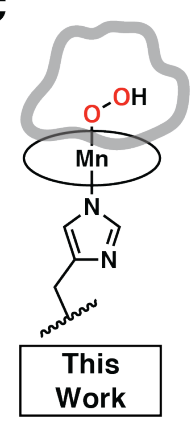

Figure 1. (A) Common metal-oxygen intermediates utilized in heme proteins. (B) Illustrations of designed function used for PS1 and (C) MPP1. PS1 was designed to undergo photophysical function, therefore no binding site access was required, and an Ile residue was placed above the distal face of the porphyrin to maximize packing with the cofactor. The binding site of MPP1 was designed with adequate space for two-O atoms above the Mn center for accessibility by using a dioxygen unit in the design. Circles represent porphyrin macrocycles.

Expanding on the design strategy for PS1 (dubbed Enfold), which simultaneously packed the binding site and folded core residues, we computationally designed a protein for a new abiological cofactor, MnDPP (Mn-diphenylporphyrin, Fig. 2A). This cofactor is larger and contains a redox active metal $(\mathrm{Mn})$ providing a good test of the breadth of our approach. The ability to access multiple redox states additionally expands the scope of possible reactivity with computationally designed proteins. Akin to native heme proteins, Mn-aryl porphyrins are capable of performing a variety of oxidative transformations, including epoxidation, sulfoxidation, and alcohol oxidation, via high-valent $\mathrm{Mn}$-intermediates (i.e. Mn(IV) or $\mathrm{Mn}(\mathrm{V})) .^{2}$ Formation of these strongly oxidizing intermediates presents additional design challenges that have not been previously implemented with precision in the de novo design of porphyrin proteins: 1) to purposefully design access to the metal center for oxidants and/or substrates and 2) to limit the ability of these powerful metal-based oxidants to deleteriously react with the protein scaffold itself. In the design of PS1, emphasis was placed on a well-folded core thereby restricting access to the cofactor. ${ }^{14}$ Natural oxidases and peroxidases often maintain access channels to the cofactor with water molecules during the resting state, which are then displaced during catalysis. ${ }^{3,21}$ We implement this concept in our design by using a dioxygen unit in the open coordination site of the Mn center. We have shown that this strategy provides access to the binding site for solvent, oxidants, and substrate and prevents detrimental damage to the binding site.
A
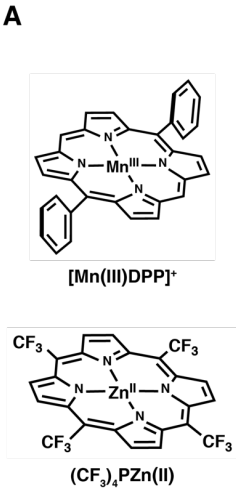

C
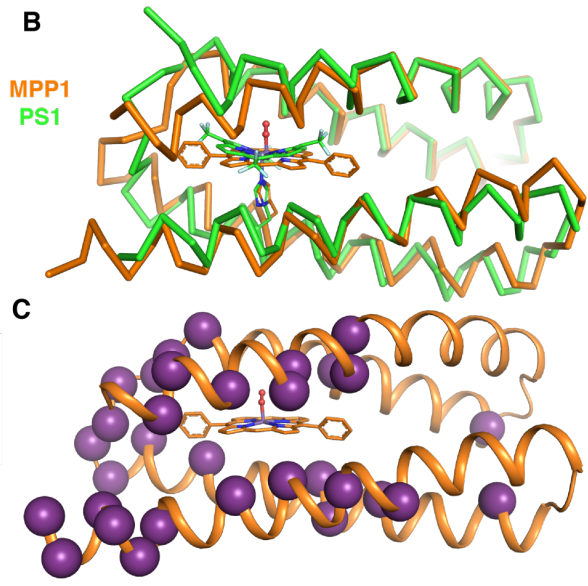

Figure 2. Comparison of MPP1 design to previously designed PS1. (A) ChemDraw representations of cofactors used for design, $[\mathrm{Mn}(\mathrm{III}) \mathrm{DPP}]^{+}$(this work) and $\left(\mathrm{CF}_{3}\right)_{4} \mathrm{PZn}(\mathrm{II})$ (previous work). (B) A ribbon representation of the MPP1 design overlaid with the NMR structure of PS1 illustrating the differences in backbone length and position. (C) A cartoon representation of the MPP1 design showing the changes in sequence relative to PS1 (purple spheres) to accommodate the new cofactor.

\section{RESULTS}

Design of MPP1. The cofactor targeted in the design was a diphenyl porphyrin, MnDPP, with a dioxygen unit bound to the metal center (Fig. 2). The inclusion of the dioxygen assures sufficient space for oxidant and substrate access to the cofactor. As compared to our earlier design of PS1, which binds to $\left(\mathrm{CF}_{3}\right)_{4} \mathrm{PZn}, \mathrm{MnDPP}$ represents a more extended cofactor with a flatter porphyrin ring. Previous proteins designed to bind diphenyl metalloporphyrins failed to result in sequences whose properties were amenable to high-resolution structure determination. ${ }^{22}$ Thus, the design and high-resolution structure determination of a MnDPP-binding protein represented a significant challenge

The design of MPP1 (Manganese Porphyrin-binding Protein 1) began with the parameterized backbone of PS $1,{ }^{14}$ which includes an axial His ligand and a second-shell $\mathrm{H}$ bonded Thr on an adjacent helix. The MnDPP was placed with its phenyl rings and the metal ion oriented along the central axis of the bundle (Fig. 2). While PS1 was used as a starting template, the bundle needed to be extended to accommodate the larger diphenylporphyrin cofactor. Thus, an idealized helical bundle was fit to the structure of PS1 and the helices were elongated to match the length of the cofactor (plus an additional region intended to form a well-packed hydrophobic core). The dioxygen unit (formally $\mathrm{OOH}^{-}$) from an oxy-P450 structure $^{23}$ (PDB 2A1M) was placed in the open coordination site on the Mn center $(\sim 1.8 \AA \mathrm{Mn}-\mathrm{O}$ distance, trans to the His ligand, Fig. 2B-C).

Using a custom RosettaScript, the interior residues were allowed to vary through iterative cycles of flexible-backbone sequence design. Due to the high expression levels and stability of PS1, most solvent exposed residues were unchanged. To minimize potential oxidation of the protein, Trp and Tyr residues were disallowed at positions within $12 \AA$ of the cofactor and Met and Cys residues were disallowed throughout during design. Models were assessed for low 
energy and good packing scores by analyzing packing score (pstat) and total energy scores from Rosetta outputs. The initial design of the helical sequences was conducted without connecting loops, because this approach provided the most flexibility to allow the protein to optimize its fit with the cofactor.
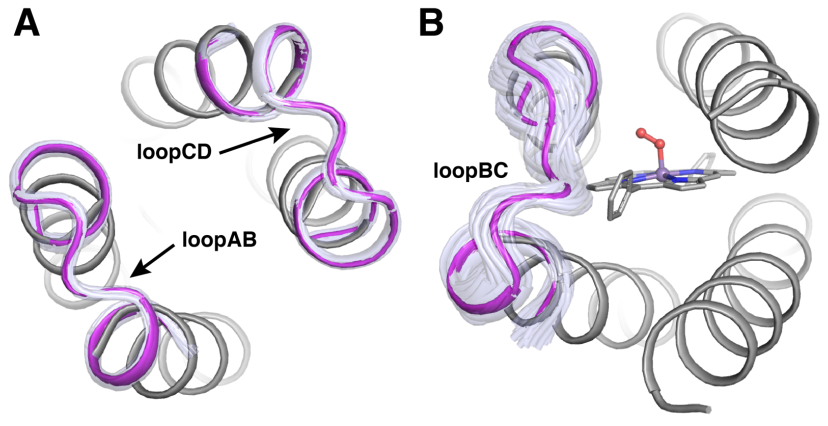

Figure 3. Design model of MPP1 showing representative clusters of loops. Clustered loops from MASTER for loopAB and loopCD (A) and loopBC (B). Loop clusters are shown in light purple and the chosen loop in magenta.

To create single-chain proteins, loops were designed using MASTER ${ }^{24}$ In MASTER an input structural element (query) is compared to a library of three-dimensional protein structures using the backbone root-mean-square deviation (RMSD) as similarity score. Library structural fragments that match the query to a given RMSD threshold are enumerated and the designability of a query is quantified by the total number of structural matches found in the library. Short segments of the two helices (6 residues) were queried against a database of helical proteins from the PDB searching for a particular loop length to connect them. This allows for the use of loops that are well represented in natural proteins and are good matches for the structural context of the designed backbone. Loop length and sequence are important parameters to consider in the design of highly stable parameterized helical backbones; ${ }^{25-26}$ therefore, loops were clustered by RMSD and clusters with highly represented loops were chosen. As a result, Helices A and B (loopAB) and C and D (loopCD) were connected with short, highly designable (as evidenced by the tight clustering) nested Schellman plus N-capping loop motifs ${ }^{25-29}$ (Fig. 3A) to ensure a stable folded core. MASTER proved particularly useful in choosing loopBC. To accommodate the larger cofactor and, as a result, longer inter-helical distance between helices B and C, a longer loop was required. Overall, these loops were less well-represented in the PDB than loops $\mathrm{AB} / \mathrm{CD}$ but a large cluster was found nonetheless (see Supporting Information, Fig. 3B, Table S1). After building loops, additional rounds of flexible backbone sequence design were performed to ensure complementary packing of the interior of the bundle with the new loops. At this stage, three addition designs (MPP2-4) were prepared with different lengths loops for loopBC, including the original loop from PS1 (Fig. S1).

PS1 showed the importance of adding Gly and Ala residues to create a more polar binding site and avoid hydrophobic collapse in the absence of the cofactor, ${ }^{14}$ as can be an issue in heme-binding maquettes. ${ }^{30-31}$ Therefore, three Gly residues were constrained in close proximity to the cofactor to ensure sufficient flexibility in the binding region for apoMPP1. Additionally, the final MPP1 sequence had seven Ala residues in the binding site. Together, these small residues were intended to enable easy access to the Mn center, and minimize the number of oxidizable bonds in close proximity to the cofactor.

Despite coming from the same parameterized backbone, $65 \%$ of the interior residues in MPP1 changed relative to PS1, with the majority of changes occurring in the binding site (Fig. 2C). Moreover, the sequence of MPP1 has no similarity with any natural protein (Blast $E$ value $<0.05$ against non-redundant protein database).

As a test of structural stability of apo-MPP1, ab initio folding predictions were run on the designed sequence. ${ }^{32}$ As expected, the binding region appears more flexible relative to the conformationally unique folded core (Fig. S2). As discussed above, the addition of Gly and Ala residues to the binding region, along with polar His and Thr residues, maintain an appropriately dynamic binding site. And the lower RMSD clustering of the results for the folded core region, relative to the binding site, suggest a level of preorganization of the distal core residues.
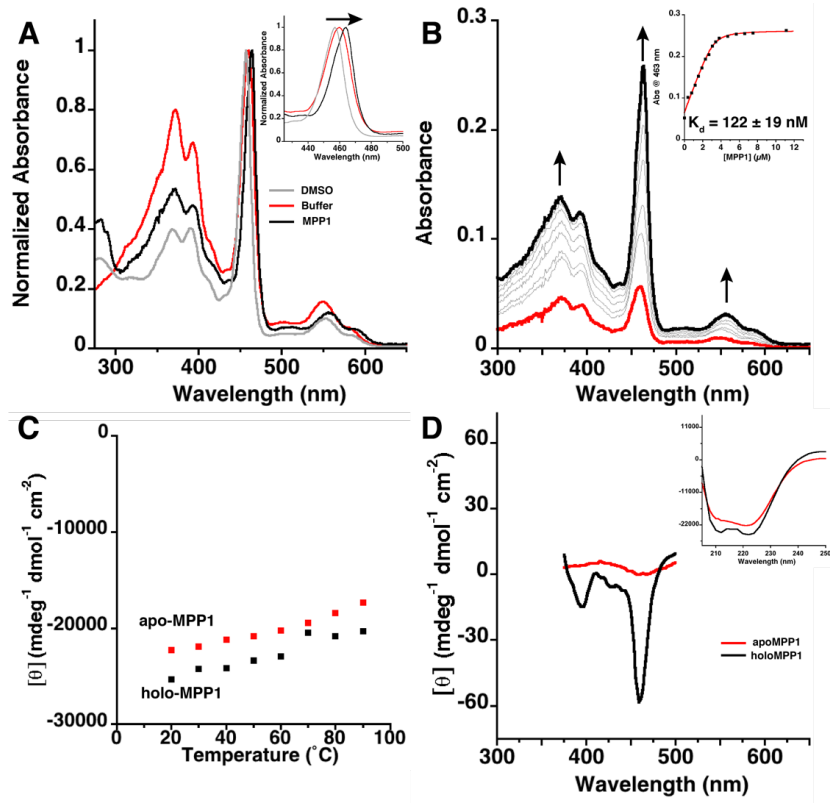

Figure 4. (A) Electronic absorption spectra showing the red shift for Mn(III)DPP Soret band in DMSO, buffer, and MPP1. Inset: Zoom in of Soret bands. (B) Electronic absorption spectra showing the titration of apo-MPP1 into a solution of Mn(III)DPP to determine $\mathrm{K}_{\mathrm{d}}$. Inset: plot of absorption at $463 \mathrm{~nm}$ versus MPP1 concentration and fit (in red). $\mathrm{K}_{\mathrm{d}}$ error is from fit. (C) Temperature dependent $\mathrm{CD}$ plot showing the thermal stability of apo- and holo-MPP1 (ellipticity measured at $222 \mathrm{~nm}$ ). (D) CD spectra in UV (inset) and visible regions of apo- and holo-MPP1 showing the induced Cotton effect. Buffer conditions were: $50 \mathrm{mM}$ MES, $150 \mathrm{mM} \mathrm{NaCl} \mathrm{pH} \mathrm{7.5.}$

Solution Characterization. MPP1 - MPP4 all express well in E.coli (20-30 mg/L) and were purified to homogeneity (Fig. S3). Spectral titrations indicated that they each bound Mn(III)DPP with similar shifts in the absorption spectrum strongly suggesting that they accommodate the cofactor in a similar binding environment (Fig. S4). As a preliminary test of unique solution structure, ${ }^{1} \mathrm{H}$ NMR spectra were collected for holo-MPP1-4 (data not shown). MPP1-2 appeared to be well structured in solution while MPP3-4 showed evidence of conformational averaging, similar to previously designed maquettes. MPP1 crystallized readily (see below), while 
MPP2 did not form crystals in preliminary crystallization trials. We therefore focused our efforts on MPP1.

MPP1 tightly binds Mn(III)DPP $\left(\mathrm{K}_{\mathrm{d}}=122 \pm 19 \mathrm{nM}\right)$

despite the cofactor's poor aqueous solubility. The cofactor was added from a DMSO stock solution and binding occurs within the mixing time, resulting in a red-shift in the Mn(III)DPP split Soret absorption manifold and Q-band transitions (Fig. 4A-B). The absorbance at $463 \mathrm{~nm}$ is consistent with binding of an axial His ligand. ${ }^{33}$ In addition, the measured extinction coefficient $\left(\boldsymbol{\varepsilon}_{463}=65,000 \mathrm{M}^{-1} \mathrm{~cm}^{-1}\right)$ of the Soret band suggests that the $\mathrm{Mn}(\mathrm{III})$ center is six-coordinate in solution, consistent with the design of His and water as axial ligands under these conditions. ${ }^{34-35}$ Although MnDPP is achiral, binding to the active site is expected to result in induced chirality if it is held rigidly within the cofactor binding site. Indeed, the visible CD spectrum of holo-MPP1 shows a clear induced Cotton effect associated with the Soret band transitions centered at 391 and $463 \mathrm{~nm}$, matching the electronic absorption spectrum (Fig. 4). It should be noted that despite starting from a parameterized PS1 helical bundle, MPP1 does not bind tightly to $\left(\mathrm{CF}_{3}\right)_{4} \mathrm{PZn}$ (Fig. S5).

MPP1 was designed to be exceptionally well-folded, leading to extreme thermal stability. This allows for ease of study as well as the possibility of doing catalytic reactions that require high temperatures. As designed, apo- and holo-MPP1 are thermostable, with $\mathrm{T}_{\mathrm{m}}>90{ }^{\circ} \mathrm{C}$ (Fig. 4C). This level of thermostability is on par (or better) with thermophilic proteins from extremophiles. In fact, the induced CD of the Soret bands are still evident at $90{ }^{\circ} \mathrm{C}$ (Fig. S6). Furthermore, the protein prevents aggregation of the Mn-cofactor which readily occurs in the absence of the protein.

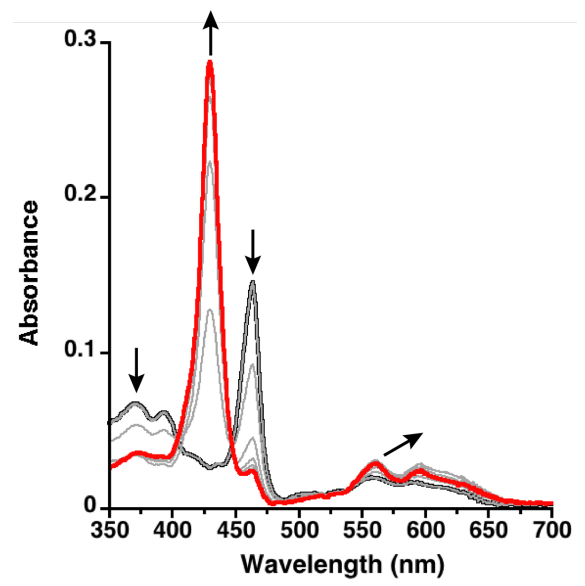

Figure 5. Spectroelectrochemical titration of Mn(III)MPP1 (black) to Mn(II)MPP1 (red) in the presence of redox mediator indigo-5,5'-disulfonate (IDIS). The potential was decreased from 10 to $-390 \mathrm{mV}$ vs SHE in $50 \mathrm{mV}$ increments, holding each potential until no further spectral changes were observed. The spectra of IDIS under the same conditions have been subtracted for clarity.

To determine the effect of the protein environment on the $\mathrm{Mn}(\mathrm{III} / \mathrm{II})$ reduction potential, spectrophotometric dithionite titration experiments were performed (see SI). ${ }^{36}$ The $\mathrm{E}_{1 / 2}\left(\mathrm{Mn}^{\mathrm{III} / \mathrm{II}}\right)$ for holo-MPP1 was found to be $-107( \pm 5) \mathrm{mV}$ vs SHE (pH 7, Fig. S7). ${ }^{15,20,37}$ Because redox-coupled protonation/deprotonation play critical roles in catalysis it was important to assess changes in the protonation state accompanying changes in the oxidation state of the Mn ion. Therefore, we confirmed and extended these results using an independent, spectroelectrochemical method that employs a true reference electrode to measure changes in $\mathrm{Mn}(\mathrm{III} / \mathrm{II})$ reduction potential as a function of $\mathrm{pH}$ (see SI, Figs. 5 and S8, Table S2). A midpoint potential of $-145 \mathrm{mV}$ vs $\mathrm{SHE}$ was observed at $\mathrm{pH} 7$, in agreement with the value measured by spectrophotometric solution titration. The observed midpoint potential is constant from $\mathrm{pH} 8$ to 7. At $\mathrm{pH}$, it became less negative by $30 \mathrm{mV}$, indicative of partial protonation of a group that is thermodynamically coupled to the $\mathrm{Mn}$ (II/III) ion (Table S2). At lower $\mathrm{pH}$, one might expect full protonation in the reduced state, and, therefore, a full $60 \mathrm{mV} / \mathrm{pH}$ would be observed, as expected for a $1 \mathrm{e}-/ 1 \mathrm{H}^{+}$process. Unfortunately, measurements at lower $\mathrm{pH}$ were complicated by possible protonation of the His ligand.

The cofactor can be reduced from $\mathrm{Mn}$ (III) to $\mathrm{Mn}$ (II) upon addition of one equivalent of dithionite to a degassed solution, causing a blue-shift in the Soret (Fig. S7). The Mn(II) species is stable in the absence of dioxygen, however after oxygenation the spectrum quantitatively evolves to that for the $\mathrm{Mn}$ (III) species indicating that (1) the binding site is accessible to dioxygen and (2) the intermediate species does not deleteriously effect the binding environment (Fig. S9).

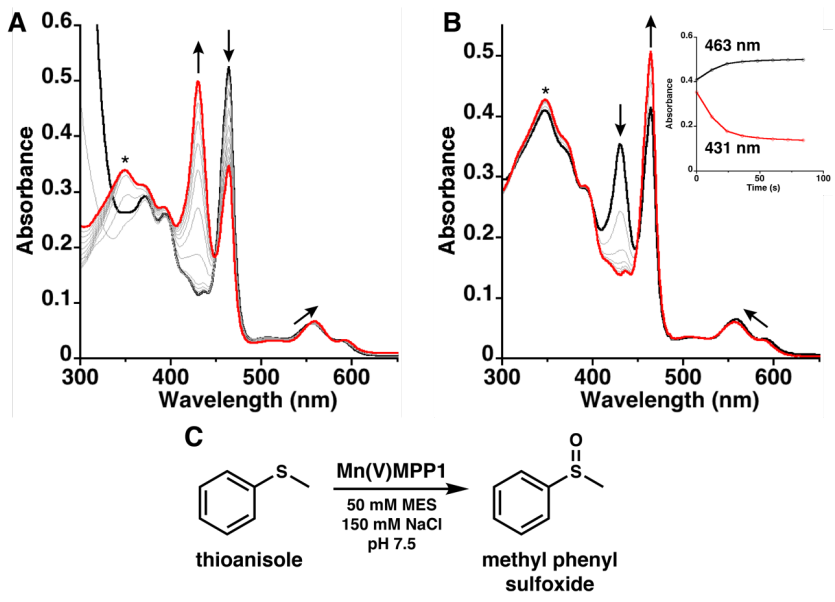

Figure 6. (A) Electronic absorption spectra showing the reaction of Mn(III)MPP1 (black) with excess $\mathrm{NaIO}_{4}$ to form a Mn(V)-oxo species monitored with 60 s scans. (B) Reaction of the $\mathrm{Mn}(\mathrm{V})$ oxo species (red) with 10 equivalents of thioanisole showing the loss of the $\mathrm{Mn}(\mathrm{V})$-oxo bands and conversion back to the Mn(III) spectrum (black) monitored with $12 \mathrm{~s}$ scans. Inset: Time trace monitoring absorbances at $463 \mathrm{~nm}$ and $431 \mathrm{~nm}$. (C) Scheme showing sulfoxidation of thioanisole. $\left(^{*}\right)$ indicates the absorbance from $\mathrm{NaIO}_{4}$ (see SI).

Formation of Mn(V) Species and Sulfoxidation Reaction. To design functional metalloproteins, there must be access to the cofactor for oxidant and substrate to reach the metal center, but the protein scaffold must also support a high-valent species without deleterious effects. Mn-porphyrin catalysts often go through a $\mathrm{Mn}(\mathrm{V})$-oxo intermediate as the competent oxidant via a 2-electron oxidation from $\mathrm{Mn}(\mathrm{III})$ to $\mathrm{Mn}(\mathrm{V}){ }^{4}$

These species have been well studied in the literature, with diagnostic absorption spectra. ${ }^{38-40}$ To test MPP1's ability to support formation of these highly-oxidizing species the $\mathrm{Mn}$ (III) holo-protein was oxidized in solution with sodium periodate $\left(\mathrm{NaIO}_{4}\right)$, a 2-electron oxidant and O-atom transfer agent. Upon addition, the Soret transition centered at $463 \mathrm{~nm}$ associated with the Mn(III) species decreases with a concomitant evolution of a band at $431 \mathrm{~nm}$ that matches the literature for similar species assigned as a Mn(V)-oxo species (Fig. 6). ${ }^{38-40}$ This reaction proceeds with approximately $50 \%$ conversion based on 


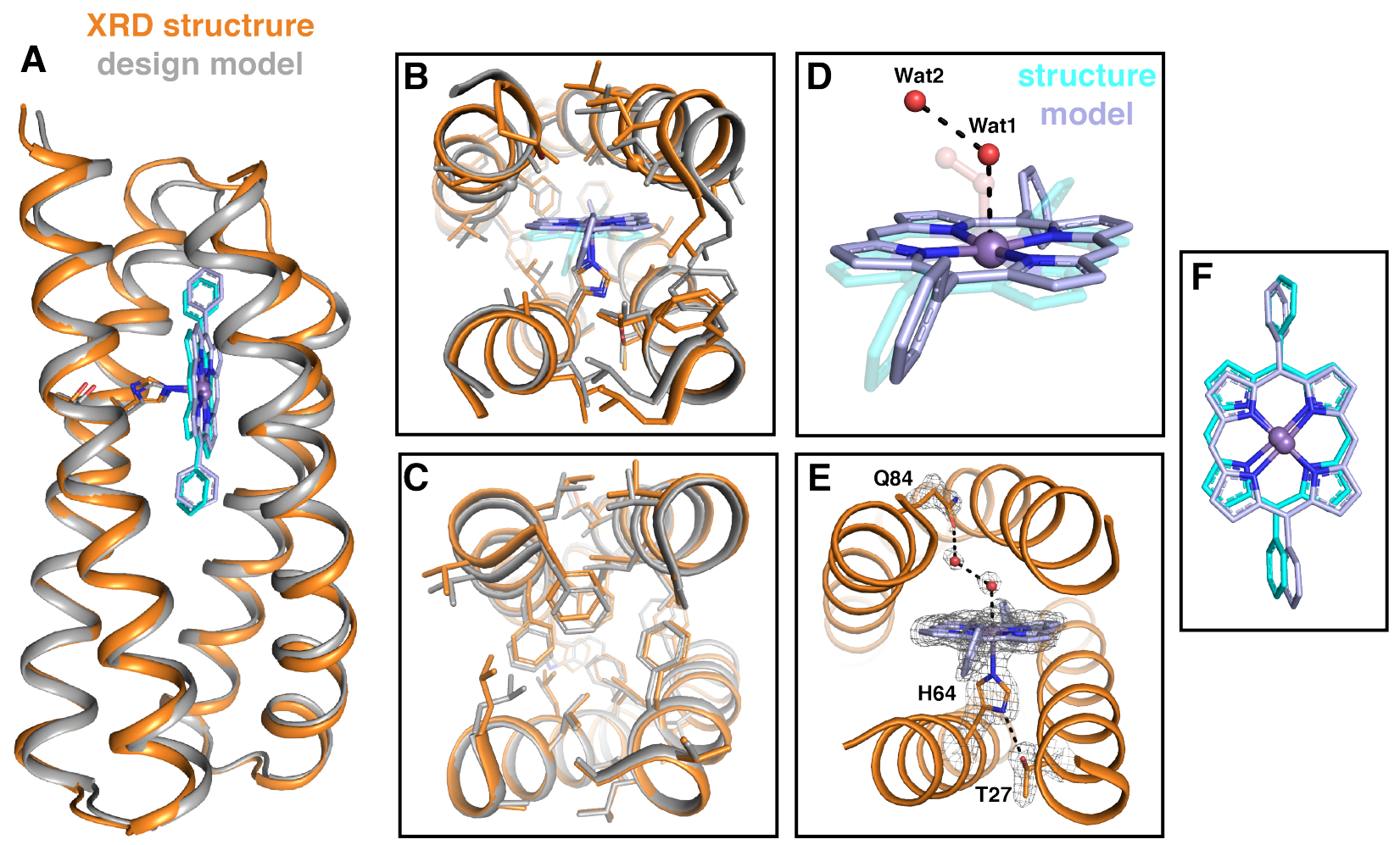

Figure 7. Structural comparison of the designed model of MPP1 (grey) and the crystal structure (PDB: 7JRQ, orange). (A) Cartoon representation showing an extremely good backbone match between the design and structure ( $0.6 \AA$ all backbone rmsd). Comparisons of the binding site region $(\mathbf{B})$ and folded core region $(\mathbf{C})$. Comparison of the placement of Wat 1 and Wat2 (red spheres) relative to the dioxygen unit in the design (transparent) (D) and extended H-bonding network from the binding site to the surface via a water network (E). The po-

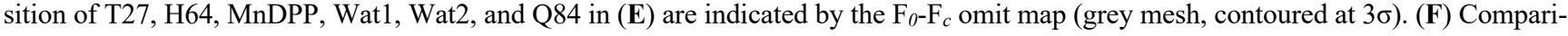
son of observed cofactor placement (grey) relative to the model (cyan). Glycine residues are shown as $\mathrm{C} \alpha$ spheres.

the absorbance of the $\mathrm{Mn}(\mathrm{III})$ species. At room temperature, the $\mathrm{Mn}(\mathrm{V})$ species is unstable and slowly converts back to the starting $\mathrm{Mn}$ (III) spectrum with a half-life of $\sim 12$ minutes (Fig. S10).

However, addition of thioanisole to the pre-formed $\mathrm{Mn}(\mathrm{V})$ species leads to a rapid decrease in the $431 \mathrm{~nm}$ band and return of the $\mathrm{Mn}$ (III) starting spectrum. Analysis of the reaction mixture by mass spectroscopy verified the formation of methyl phenyl sulfoxide with no evidence for further oxidation to phenyl methyl sulfone (Fig. 6C and S11). This sulfoxidation reaction is consistent with the assignment of a $\mathrm{Mn}(\mathrm{V})$-oxo species forming upon addition of periodate to the $\mathrm{Mn}$ (III) holo-protein. ${ }^{41-42}$ It should be noted that in the absence of the protein, the cofactor does not form this species and, instead, slowly decomposes in the presence of $\mathrm{NaIO}_{4}$ (Fig. S12) strongly suggesting that the protein environment helps stabilize this species.

Structural Characterization. Holo-MPP1 is the first crystallographically characterized de novo designed porphyrin-binding protein, a significant step forward in understanding how to build binding sites to create functional proteins. The structure was solved to $1.75 \AA$ resolution to reveal that the backbone matches the design exceptionally well $(0.6$ $\AA$ all backbone RMSD, Fig. 7). LoopBC is well-structured, however it is displaced relative to the design, likely because of an N-terminal 6-His tag and TEV cleavage sites. This tag, which was not included in the design, interacts with the loopBC in the crystal structure (Fig. 7A and S13). The protein crystallized with an additional MnDPP unit at a symmetry interface on the surface bound to E58 (see SI, Fig. S14). However, in aqueous solution, the protein is monomeric and binds a single equivalent of cofactor (Fig. 4B and S3). Thus, the additional inter-subunit cofactor must only assemble under the crystallization conditions.

The His-bound cofactor sits precisely where it was placed in the design, predicting the Mn position within $0.5 \AA$, less than the length of a $\mathrm{C}-\mathrm{H}$ bond. The only difference is a $55^{\circ}$ rotation in the torsion angle of one of the macrocycle's meso-phenyl groups (Fig. 7). In addition to the extraordinary similarity between the structure and design, the structure confirmed Mn-His coordination and revealed two water molecules (Wat1 and Wat2) within the binding site (Fig. 7). Wat1 is a weakly coordinating sixth ligand to the Mn center (Wat1-Mn distance 2.62(1) $\AA$ ), which agrees with the solution absorption spectrum of MPP1 and matches the design. The Wat1-Mn distance is similar to the $\mathrm{Mn}-\mathrm{O}$ (water) distance found in a Mnporphyrinoid substituted myoglobin. ${ }^{43}$ Wat2 forms an H-bonding network that includes Wat 1 and Gln84. As expected, the Wat1-Mn and Wat1-Wat2 distances are longer than the analogous distances in the design because they are not part of a covalent dioxygen unit, and rather a H-bonded network. However, the two water molecules do sit along the same vector as the $\mathrm{O}-\mathrm{O}$ bond in the design with an identical $\mathrm{Mn}-\mathrm{O}-\mathrm{O}$ angle of $124^{\circ}$ (Fig. 7D). As in natural proteins, these water molecules within the binding site may act as a placeholder to maintain an access channel. This is the first structural evidence of binding site access for a designed porphyrin-binding protein, a major advance towards the development of functional metalloproteins from scratch, and, we believe, a result of purposefully designed access using a dioxygen unit.

\section{DISCUSSION}


The long history of de novo designed porphyrinbinding proteins has taught us much, but the ability to gain structural insight is invaluable. As the first crystallographically characterized de novo designed porphyrin-binding protein, MPP1 illustrates the importance in determining the protein structure to further our understanding of the design features necessary to produce functional proteins from scratch. This work expands on the Enfold ${ }^{14}$ strategy to include purposefully designed substrate access by including a two $\mathrm{O}$-atom unit within the binding site. Additionally, it demonstrates the generality of this approach - all parts of the protein are important: a well-folded distal core, shape complimentary binding site, and appropriate space reserved for external access. When designed in unison, the resulting protein structure was highly predictable without the need for evolution or experimental optimization. All four sequences designed bound the cofactor with high affinity, although two showed evidence of conformational averaging in solution. Of the two that showed an NMR spectrum consistent with a unique structure, one was crystallized and structurally interrogated.

The structure showed the presence of two water molecules in an H-bonding network that sit above the Mn proximal to the designed location of the dioxygen unit. This finding suggests that MPP1, as designed, is capable of accommodating two O-atom units during activation of dioxygen. Moreover, the designed metalloprotein can access a $\mathrm{Mn}(\mathrm{V})$-oxo species and perform $\mathrm{O}$-atom transfer to thioanisole. With the structure of MPP1 in hand, we can redesign the binding site to include an active site customized for substrate. This work is a substantial step forward in protein design and paves the way for functional metalloproteins designed from scratch.

\section{METHODS}

Protein Design. Full methods and scripts regarding the design of MPP1 are given in the Supporting Information. Briefly, the parameterized backbone of PS1 was re-designed to bind Mn(III)DPP with a dioxygen unit bound in the open coordination site via a customized Rosetta script for flexible-backbone sequence design. The flexible backbone protocol was: distance and angle constraints between $\mathrm{Mn}$ and His were loaded, the model was repacked, the backbone and then side chains were relaxed, three trials of a Monte Carlo flexible backbone sub-protocol were performed and filtered for models with native-like packing (pstat $\geq 0.48$ ). Models were analyzed for packing and total energy. The designs of MPP2-4 proceeded in the same fashion.

Protein expression and purification. Full details are given in the Supporting Information. Briefly, the gene coding for the protein sequence of MPP1 was ordered from IDT, which was cloned into the IPTG-inducible pET-21a plasmid (cloning site NdeI-BamHI). The sequence also coded for an N-terminal 6xHis-tag followed by a TEV protease cleavage sequence, followed by the designed sequence. Porphyrin binding titration to determine $\boldsymbol{K}_{\mathrm{D}}$. $\mathrm{Mn}(\mathrm{III}) \mathrm{DPP}(\mathrm{Cl})$ was purchased from Frontier Scientific and used as received. 3.4 $\mu \mathrm{M}$ of $\mathrm{Mn}(\mathrm{III}) \mathrm{DPP}(\mathrm{Cl})$ was solubilized in a $1 \mathrm{~mL}$ solution of $50 \mathrm{mM}$ MES, $150 \mathrm{mM} \mathrm{NaCl}, \mathrm{pH} 7.5$ buffer from a DMSO stock solution. $1 \mu \mathrm{L}$ of a $308 \mu \mathrm{M}$ stock of apo-MPP1 was titrated into the $1 \mathrm{~mL}$ solution containing the porphyrin, and an electronic absorption spectrum was measured until $>2.5$ equivalents of protein were added. Absorbance changes at $463 \mathrm{~nm}$, due to His-Mn coordination-induced spectral shifts of the porphyrin, were fit to a single-site, protein-ligand binding model.

Preparation of holo-MPP1. 1.2 equivalents $(60 \mu \mathrm{L})$ of a $10 \mathrm{mM}$ DMSO solution of $\mathrm{Mn}(\mathrm{III}) \mathrm{DPP}(\mathrm{Cl})$ was added to $1 \mathrm{~mL}$ of a $500 \mu \mathrm{M}$ apo-MPP1 solution in $50 \mathrm{mM}$ MES, $150 \mathrm{mM} \mathrm{NaCl} \mathrm{pH} 7.5$ and allowed to sit for 5 minutes at room temperature. The protein/cofactor solution was centrifuged at $14,000 \mathrm{~g}$ in an Amicon Ultra $-0.5 \mathrm{~mL}$ centrifuge filter (10 kDa MW cutoff) for 3 minutes, 3 times, replacing the buffer to $0.5 \mathrm{~mL}$ after each spin. Finally, the solution was spun for 2 minutes at $12,000 \mathrm{~g}$ in an Amicon ultrafree-MC GV filter. The holoMPP1 was stored at $4{ }^{\circ} \mathrm{C}$ for short-term storage or $-20{ }^{\circ} \mathrm{C}$ for longterm storage. Binding tests of $\left(\mathrm{CF}_{3}\right)_{4} \mathrm{PZn}$ were carried out with the same method.

\section{ASSOCIATED CONTENT}

\section{Supporting Information}

The Supporting Information is available free of charge on the ACS Publications website.

Additional methods for protein design, spectroelectrochemistry, $\mathrm{Mn}(\mathrm{V})$ formation and reactivity, and crystallography, Figures S1S14, and Tables S1-S3 (PDF)

\section{AUTHOR INFORMATION}

\author{
Corresponding Author \\ william.degrado@ucsf.edu
}

\section{ORCID}

Samuel I. Mann: 0000-0001-8358-7182 Animesh Nayak: 0000-0001-7251-7592

George T. Gassner: 0000-0002-7359-6699

Michael J. Therien: 0000-0003-4876-0036

William F. DeGrado: 0000-0003-4745-263X

\section{Notes}

The authors declare no competing financial interest. Atomic coordinates for holo-MPP1 have been deposited in the Protein Data Bank with accession number: 7JRQ.

\section{ACKNOWLEDGMENT}

The authors thank Dr. Yibing Wu for recording and analyzing NMR data, Dr. Nick Polizzi for helpful discussions about Rosetta protocols, and Dr. Jack Nicoludis for helpful discussions about XRD data processing. We would also like to thank A.S. Borovik and Dr. Kelsey Miller for allowing us to use their synchrotron beamtime. X-ray diffraction experiments were performed at the Stanford Synchrotron Radiation Light Source on beamline 12-2. This work was primarily supported by NIGMS F32 GM130029, NSF grants 1709506 and CHE-1709497, and AFOSR FA9550-19$1-0331$.

\section{ABBREVIATIONS}

DPP, Diphenylporphyrin; MPP1-4, Manganese Porphyrin Binding Protein.

\section{REFERENCES}

1. Reedy, C. J.; Gibney, B. R., Heme Protein Assemblies. Chem. Rev. 2004, 104 (2), 617-650.

2. Huang, X.; Groves, J. T., Oxygen Activation and Radical Transformations in Heme Proteins and Metalloporphyrins. Chem. Rev. 2018, 118 (5), 2491-2553.

3 . Poulos, T. L., Heme Enzyme Structure and Function. Chem. Rev. 2014, 114 (7), 3919-3962.

4. Baglia, R. A.; Zaragoza, J. P. T.; Goldberg, D. P., Biomimetic Reactivity of Oxygen-Derived Manganese and Iron Porphyrinoid Complexes. Chem. Rev. 2017, 117 (21), 13320-13352.

5. Lyaskovskyy, V.; Suarez, A. I.; Lu, H.; Jiang, H.; Zhang, X. P.; de Bruin, B., Mechanism of cobalt(II) porphyrin-catalyzed C-H amination with organic azides: radical nature and $\mathrm{H}$-atom abstraction ability of the key cobalt(III)-nitrene intermediates. J. Am. Chem. Soc. 2011, 133 (31), 12264-73. 
6. Chen, K.; Arnold, F. H., Engineering new catalytic activities in enzymes. Nat. Catal. 2020, 3 (3), 203-213.

7. Brandenberg, O. F.; Fasan, R.; Arnold, F. H., Exploiting and engineering hemoproteins for abiological carbene and nitrene transfer reactions. Curr. Opin. Biotechnol. 2017, 47, 102-111.

8. Korendovych, I. V.; DeGrado, W. F., De novo protein design, a retrospective. Q. Rev. Biophys. 2020, 53, e3.

9. $\quad$ Pinter, T. B. J.; Koebke, K. J.; Pecoraro, V. L., Catalysis and Electron Transfer in De Novo Designed Helical Scaffolds. Angew. Chem., Int. Ed. Engl. 2020, 59 (20), 7678-7699.

10. Watkins, D. W.; Armstrong, C. T.; Anderson, J. L., De novo protein components for oxidoreductase assembly and biological integration. Curr. Opin. Chem. Biol. 2014, 19, 90-8.

11. Huang, P. S.; Boyken, S. E.; Baker, D., The coming of age of de novo protein design. Nature 2016, 537 (7620), 320-7.

12. Cheng, R. P., Beyond de novo protein design--de novo design of non-natural folded oligomers. Curr. Opin. Struct. Biol. 2004, 14 (4), 512-20.

13. Lombardi, A.; Pirro, F.; Maglio, O.; Chino, M.; DeGrado, W. F., De Novo Design of Four-Helix Bundle Metalloproteins: One Scaffold, Diverse Reactivities. Acc. Chem. Res. 2019, 52 (5), 11481159.

14. Polizzi, N. F.; Wu, Y.; Lemmin, T.; Maxwell, A. M.; Zhang, S.-Q.; Rawson, J.; Beratan, D. N.; Therien, M. J.; DeGrado, W. F., De novo design of a hyperstable non-natural protein-ligand complex with sub-Å accuracy. Nat. Chem. 2017, 9 (12), 1157-1164.

15. Farid, T. A.; Kodali, G.; Solomon, L. A.; Lichtenstein, B. R.; Sheehan, M. M.; Fry, B. A.; Bialas, C.; Ennist, N. M.; Siedlecki, J. A.; Zhao, Z.; Stetz, M. A.; Valentine, K. G.; Anderson, J. L. R.; Wand, A. J.; Discher, B. M.; Moser, C. C.; Dutton, P. L., Elementary tetrahelical protein design for diverse oxidoreductase functions. Nat. Chem. Biol. 2013, 9 (12), 826-833.

16. Koder, R. L.; Anderson, J. L.; Solomon, L. A.; Reddy, K. S.; Moser, C. C.; Dutton, P. L., Design and engineering of an $\mathrm{O}(2)$ transport protein. Nature 2009, 458 (7236), 305-9.

17. Kamtekar, S.; Schiffer, J. M.; Xiong, H.; Babik, J. M.; Hecht, M. H., Protein design by binary patterning of polar and nonpolar amino acids. Science 1993, 262 (5140), 1680-5.

18. Moffet, D. A.; Foley, J.; Hecht, M. H., Midpoint reduction potentials and heme binding stoichiometries of de novo proteins from designed combinatorial libraries. Biophys. Chem. 2003, 105 (2-3), 231-239.

19. Moffet, D. A.; Certain, L. K.; Smith, A. J.; Kessel, A. J.; Beckwith, K. A.; Hecht, M. H., Peroxidase Activity in Heme Proteins Derived from a Designed Combinatorial Library. J. Am. Chem. Soc. 2000, 122 (31), 7612-7613.

20. Shifman, J. M.; Gibney, B. R.; Sharp, R. E.; Dutton, P. L., Heme redox potential control in de novo designed four-alpha-helix bundle proteins. Biochemistry 2000, 39 (48), 14813-21.

21. Sevrioukova, I. F.; Poulos, T. L., Understanding the mechanism of cytochrome P450 3A4: recent advances and remaining problems. Dalton Trans. 2013, 42 (9), 3116-26.

22. Cochran, F. V.; Wu, S. P.; Wang, W.; Nanda, V.; Saven, J. G.; Therien, M. J.; DeGrado, W. F., Computational De Novo Design and Characterization of a Four-Helix Bundle Protein that Selectively Binds a Nonbiological Cofactor. J. Am. Chem. Soc. 2005, 127 (5), 1346-1347.

23. Nagano, S.; Poulos, T. L., Crystallographic study on the dioxygen complex of wild-type and mutant cytochrome P450cam Implications for the dioxygen activation mechanism. J. Biol. Chem. 2005, 280 (36), 31659-31663.

24. Zhou, J.; Grigoryan, G., Rapid search for tertiary fragments reveals protein sequence-structure relationships. Protein Sci. 2015, 24 (4), 508-24.

25. Engel, D. E.; DeGrado, W. F., Alpha-alpha linking motifs and interhelical orientations. Proteins 2005, 61 (2), 325-37.

26. Lahr, S. J.; Engel, D. E.; Stayrook, S. E.; Maglio, O.; North, B.; Geremia, S.; Lombardi, A.; DeGrado, W. F., Analysis and design of turns in alpha-helical hairpins. J. Mol. Biol. 2005, 346 (5), 1441-54.

27. Sagermann, M.; Martensson, L. G.; Baase, W. A.; Matthews, B. W., A test of proposed rules for helix capping: implications for protein design. Protein Sci. 2002, 11 (3), 516-21.

28. Schellman, C., The $\alpha$ L conformation at the ends of helices. Elsevier: New York, 1980.

29. Hovmoller, S.; Zhou, T.; Ohlson, T., Conformations of amino acids in proteins. Acta. Crystallogr. D Biol. Crystallogr. 2002, 58 (Pt 5), 768-76.

30. Skalicky, J. J.; Gibney, B. R.; Rabanal, F.; Bieber Urbauer, R. J.; Dutton, P. L.; Wand, A. J., Solution Structure of a Designed Four- $\alpha$-Helix Bundle Maquette Scaffold. J. Am. Chem. Soc. 1999, 121 (21), 4941-4951.

31. Huang, S. S.; Koder, R. L.; Lewis, M.; Wand, A. J.; Dutton, P. L., The HP-1 maquette: From an apoprotein structure to a structured hemoprotein designed to promote redox-coupled proton exchange. Proc. Natl. Acad. Sci. U. S. A. 2004, 101 (15), 5536-5541.

32. Bradley, P.; Misura, K. M.; Baker, D., Toward highresolution de novo structure prediction for small proteins. Science 2005, 309 (5742), 1868-71.

33. Yonetani, T.; Asakura, T., Comparison of manganese porphyrin-containing cytrochrome $\mathrm{c}$ peroxidase, horseradish peroxidase, and myoglobin. J. Biol. Chem. 1969, 244, 4580-4588.

34. Williamson, M. M.; Hill, C. L., Isolation and characterization of a five-coordinate manganese(III) porphyrin cation. Crystal and molecular structure of aquo(tetraphenylporphinato)manganese(III) triflate. Inorg. Chem. 1986, 25 (26), 4668-4671.

35. Hill, C. L.; Williamson, M. M., Structural and electronic properties of six-coordinate manganese(III) porphyrin cations. Crystal and molecular structure of bis $(\mathrm{N}, \mathrm{N}-$

dimethylformamide)(tetraphenylporphinato)manganese(III) perchlorate, [MnIII TPP(DMF)2]+ClO4. Inorg. Chem. 1985, 24 (18), 2836-2841.

36. Gassner, G. T., The styrene monooxygenase system. Method. Enzymol. 2019, 620, 423-453.

37. Lin, R.; Immoos, C. E.; Farmer, P. J., Unusual voltammetry of manganese-substituted myoglobin in surfactant film: evidence for two redox pathways. J. Biol. Inorg. Chem. 2000, 5 (6), 738-47.

38. Jin, N.; Groves, J. T., Unusual Kinetic Stability of a Ground-State Singlet Oxomanganese(V) Porphyrin. Evidence for a Spin State Crossing Effect. J. Am. Chem. Soc. 1999, 121 (12), 2923 2924.

39. Groves, J. T.; Lee, J.; Marla, S. S., Detection and Characterization of an Oxomanganese(V) Porphyrin Complex by Rapid-Mixing Stopped-Flow Spectrophotometry. J. Am. Chem. Soc. 1997, 119 (27), 6269-6273.

40. Nam, W.; Kim, I.; Lim, M. H.; Choi, H. J.; Lee, J. S.; Jang, H. G., Isolation of an oxomanganese(V) porphyrin intermediate in the reaction of a manganese(III) porphyrin complex and $\mathrm{H} 2 \mathrm{O} 2$ in aqueous solution. Chem.-Eur. J. 2002, 8 (9), 2067-2071.

41. Prokop, K. A.; Neu, H. M.; de Visser Sam, P.; Goldberg, D. P., A Manganese(V)-Oxo $\pi$-Cation Radical Complex: Influence of One-Electron Oxidation on Oxygen-Atom Transfer. J. Am. Chem. Soc. 2011, 133 (40), 15874-15877.

42. Mandimutsira, B. S.; Ramdhanie, B.; Todd, R. C.; Wang, H.; Zareba, A. A.; Czernuszewicz, R. S.; Goldberg, D. P., A stable manganese(V)-oxo corrolazine complex. J. Am. Chem. Soc. 2002, 124 (51), 15170-1.

43. Oohora, K.; Kihira, Y.; Mizohata, E.; Inoue, T.; Hayashi, T., C(sp3)-H bond hydroxylation catalyzed by myoglobin reconstituted with manganese porphycene. J. Am. Chem. Soc. 2013, 135 (46), 17282-5. 